\title{
A rare case of spontaneous spinal ventral extradural hematoma in a pregnant woman: Case report and review of literature
}

Sir,

Spontaneous spinal extradural hematoma (SSEH) is a rare devastating pathology, accounts about $0.3-0.9 \%$ of all epidural space-occupying lesions with a tendency to affect males, especially in the $4^{\text {th }}$ to $5^{\text {th }}$ decades. ${ }^{[1]}$ It is extremely unusual in pregnant women mainly affecting dorsal epidural region of upper thoracic region in pregnancies of 30-40 weeks gestation. ${ }^{[1]}$ Spontaneous spinal ventral extradural hematoma (SSVEH) is extremely rare event. We here introduced such a case. A 25-year-old pregnant woman week 28, G1P0, presented with sudden onset severe lower extremities weakness since $48 \mathrm{hr}$ before admission. Neurologic examination realized flaccid complete paraplegia and T4 sensory level on admission. No evidence of acute or chronic infection was detected. T2-weighted imaging (WI) on (1.5 Tesla magnetic resonance imaging) of the cervico-dorsal spine revealed a longitudinal heterogeneous hypersignal lesion in the ventral aspect of spinal epidural region at C8-T4 level with compressive effect on spinal cord [Figure 1a and c]. T1-WI disclosed mainly iso-hypointense appearance [Figure $1 \mathrm{~b}$ and $\mathrm{d}$ ]. A diagnosis of SSVEH in hyperacute-acute stage was made. Complete historical and clinical evaluation realized no predisposing factor. Due to fetal considerations, the patient's baby was delivered by cesarean section without any complication. Thereafter, spinal epidural hematoma was evacuated with posterior laminectomy approach. Follow-up selective spinal angiography was normal. Unfortunately, no clinical improvement was seen during 4 months of follow-up. Complete historical and clinical evaluation realized no predisposing factor. Our analyzing of previous pregnancy-related SSEH disclosed that SSEH mainly involves women at gestational age of 30-40. ${ }^{[2]}$ No predisposing factor has been related other than two cases who developed SEH in a setting of low molecular weight heparin consumption and one, during postpartum period. ${ }^{[2]}$ Upper thoracic epidural space (more than 70\%) especially dorsal plane is the main location involves in SSEH. ${ }^{[1]}$ More distinguished venous plexus in dorsal epidural space of thoracic region persuades some investigators to suggest that SSEH happens secondary to pregnancy-induced elevated venous pressure. ${ }^{[3]}$ This hypothesis is supported by the fact that the presence of fat as the main structure of epidural space in dorsal plane weakens it in compare to ventral plane in which tight adherence of posterior longitudinal ligament to anterior

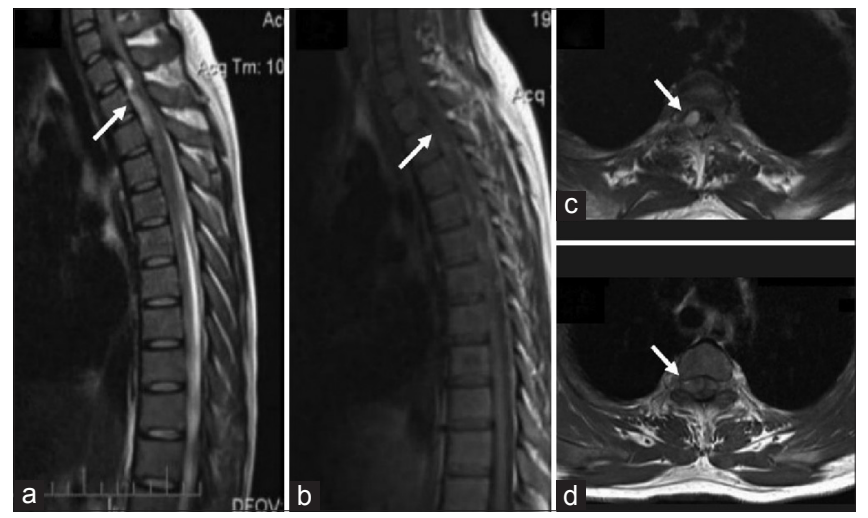

Figure 1: Sagittal and axial T2-weighted ( $a$ and c) magnetic resonance images showing a longitudinal heterogeneous hypersignal lesion in the ventral plan of epidural space extending from C8-T4 level, compressing the spinal cord (arrows). The lesion appeared mainly iso-hypointense on T1-weighted ( $b$ and $d$ ) images. Above findings are compatible with hyperacute-acute spinal epidural hematoma

dura protects it against dynamic or static traumas so far only a few cases of ventral epidural hematoma have been reported. ${ }^{[1,3]}$

In-time emergent multidisciplinary intervention is needed for minimizing neurological disabilities. ${ }^{[1]}$ Treatment is dependent to gestational age and neurological deficits. Patients with mild neurological deficits and small epidural hematoma may be managed conservatively especially when an aggravating factor is not detected. ${ }^{[1]}$ If clinical syndrome shows a progressive course, an emergent intervention will be necessary. Although previous reports showed no strong correlation between time of the symptom's onset and surgical intervention, it has been recommended that decompression should be done as soon as possible, preferentially $<12 \mathrm{~h}$ of the beginning of symptoms. Obstetrics consideration is another important factor in therapeutic plan. Cesarean section is the preferred method of delivery which commonly is advised as the first procedure in full term gestation. ${ }^{[4]}$ Due to increasing effect of uterine contraction on spinal venous pressure; vaginal delivery should be avoided. ${ }^{[1,4]}$ In early stages of gestational period, hematoma evacuation may be considered at first. If fetus is viable, pregnancy will continued and terminated with cesarean section as the fetus became mature. ${ }^{[1,3]}$

To conclude, although SSEH is an extremely rare event in pregnancy, it can be associated with severe catastrophic burden. Hence; neurologists/neurosurgeons must be 
mindful of clinical and radiological features of disease for preventing irrecoverable devastating sequels.

\section{Bahaadin Siroos, Farzad Tajik¹, Majid Ghaffarpour}

Departments of Neurology and ${ }^{1}$ Neurosurgery, Imam Khomeini Hospital, Tehran University of Medical Sciences, Tehran, Iran E-mail: ghaffarpour@sina.tums.ac.ir

\section{REFERENCES}

1. Henry JB, Messerer M, Thomas V, Diabira S, Morandi X, Hamlat A. Nontraumatic spinal epidural hematoma during pregnancy: Diagnosis and management concerns. Spinal Cord 2012;50:655-60.

2. Liu Z, Jiao Q, Xu J, Wang X, Li S, You C. Spontaneous spinal epidural hematoma: Analysis of 23 cases. Surg Neurol 2008;69:253-60.
3. Badar F, Kirmani S, Rashid M, Azfar SF, Yasmeen S, Ullah E. Spontaneous spinal epidural hematoma during pregnancy: A rare obstetric emergency. Emerg Radiol 2011;18:433-6.

4. Jo YY, Lee D, Chang YJ, Kwak HJ. Anesthetic management of a spontaneous spinal-epidural hematoma during pregnancy. Int J Obstet Anesth 2012;21:185-8.

\begin{tabular}{|l|l|}
\hline \multicolumn{2}{|c|}{ Access this article online } \\
\hline Quick Response Code: & Website: \\
\hline & www.ijns.in \\
\hline
\end{tabular}

\section{"Quick Response Code" link for full text articles}

The journal issue has a unique new feature for reaching to the journal's website without typing a single letter. Each article on its first page has a "Quick Response Code". Using any mobile or other hand-held device with camera and GPRS/other internet source, one can reach to the full text of that particular article on the journal's website. Start a QR-code reading software (see list of free applications from http://tinyurl.com/ yzlh2tc) and point the camera to the QR-code printed in the journal. It will automatically take you to the HTML full text of that article. One can also use a desktop or laptop with web camera for similar functionality. See http://tinyurl.com/2bw7fn3 or http://tinyurl.com/3ysr3me for the free applications. 